\section{Challenges to achieving clinical transplantation tolerance}

\author{
Alan D. Salama, ${ }^{1,2}$ Giuseppe Remuzzi, ${ }^{3}$ \\ William E. Harmon, ${ }^{2}$ and \\ Mohamed H. Sayegh ${ }^{1,2}$ \\ ${ }^{1}$ Laboratory of Immunogenetics and Transplantation, Renal Division, \\ Brigham and Women's Hospital, and \\ ${ }^{2}$ Nephrology Division, Children's Hospital, Harvard Medical School, \\ Boston, Massachusetts, USA \\ ${ }^{3}$ Mario Negri Institute for Pharmacological Research, Bergamo, Italy \\ Address correspondence to: Mohamed H. Sayegh, \\ Brigham and Women's Hospital, 75 Francis Street, \\ Boston, Massachusetts 02115, USA. \\ Phone: (617) 732-5259; Fax: (617) 732-5254; \\ E-mail: msayegh@rics.bwh.harvard.edu.
}

J. Clin. Invest. 108:943-948 (2001). DOI:10.1172/JCI200114142.

Transplantation is now firmly established as the therapy of choice for end-stage organ failure. Specific immunological tolerance of transplant recipients toward their foreign organ or tissue grafts is a goal that has been sought by transplant biologists for almost fifty years following the original description of the phenomenon in experimental animals (1). Since that time, a wealth of experimental data has accumulated relating to strategies for extending allograft survival and function. Still, the question remains of how near we are to the day when long-term tolerance of engrafted organs or tissues is a clinical reality.

With the pharmacopoeia of the transplant biologist continually expanding (2), the potential treatment combinations have become baffling and their impact on strategies to induce tolerance ever more complex. The increasing demand for organ transplantation and the imbalance with the supply of donor organs (3) makes it an urgent necessity to optimize the outcome of clinical transplantation. It is therefore timely to reassess where we stand on the road to achieving clinical transplant tolerance, and highlight the challenges that face us, so that we may choose the best direction in which to invest our efforts in basic and clinical research (4).

\section{A plea for tolerance}

Powerful new immunosuppressive agents, now in widespread use, have been employed in transplantation as well as in certain allergic and autoimmune conditions. These agents include humanized anti-IL-2 receptor mAb's; tacrolimus, a drug related to cyclosporin that inhibits calcineurin and blocks IL-2-dependent $\mathrm{T}$ cell activation; mycophenolate mofetil, which blocks lymphocyte purine biosynthesis; and rapamycin, an inhibitor of multiple kinase-dependent cell cycle regulators. While the use of these agents has undoubtedly improved the short-term graft survival times (2), their impact on long-term outcome remains unknown (5). Some of these treatments may indeed improve longterm kidney graft survival (6), but others are difficult to judge because they have been used clinically for too short a period (7). The improved outcome comes at a cost, with increased frequencies of malignancies and infections, as well as the individual drug-related adverse effects such as hypercholesterolemia and diabetes (2), which in turn contribute to ischemic heart disease, the cause of death of half of the transplant patients (8).

Recent trends in long-term survival rates indicate a progressive improvement of renal allograft half-lives, even before the new immunosuppressive drugs in clinical use were introduced (9). Interestingly, this improvement was only observed in patients who never had an acute rejection episode. These data emphasize the critical role of the recipient's alloimmune response as a major determinant of transplant outcome and highlight the need to develop novel strategies targeted at induction of immunologic donor-specific tolerance. Furthermore, the shortage of donor organs means that each graft should ideally be sufficient to provide lifelong replacement of function. Achieving long-term, drug-free graft acceptance with normal organ function is thus of paramount importance. It has tremendous medical and financial implications for patients with end-stage organ failure and for the health care industry as a whole. Moreover, the development of practical tolerance-inducing strategies for allotransplantation could well prove valuable in other clinical contexts, particularly autoimmune diseases and allergies.

\section{Regulation of alloreactive $T$ cells}

The key orchestrators of the immune response are the $T$ cells which can react to alloantigen both directly, by recognizing intact foreign MHC molecules on donor antigen-presenting cells (APCs), and indirectly, as a result of interactions with processed donor antigens on self APCs (10). The indirect route represents the physiologic pathway of immune recognition of foreign antigens and autoantigens.

Individual $T$ cells reacting to their specific antigen can undergo a number of different responses (Figure 1), and the factors determining the fate of a particular $\mathrm{T}$ cell are still poorly understood. However, the combination of responses by graft-specific alloreactive $\mathrm{T}$ cells will largely determine the outcome of a graft. T cells can be physiologically silenced by a number of mechanisms, including deletion, which may occur within the thymus or in the peripheral immune system; anergy, where they cannot adequately respond following restimulation with antigen; and suppression, which may be mediated by 


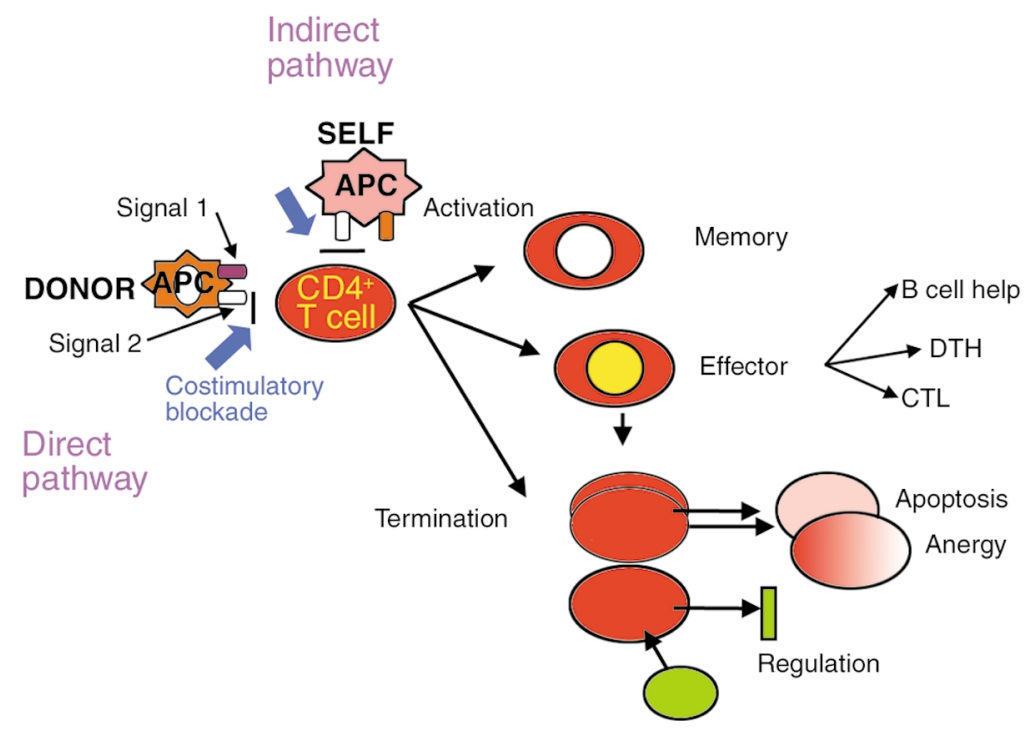

Figure 1

The fate of alloreactive T cells. Upon antigen engagement and in the presence of adequate costimulatory signals, the alloreactive $T$ cell becomes activated, proliferates, and is subject to a number of different fates. The first is differentiation to an effector cell, orchestrating the immune response directed toward the target antigen. Some of the T cells will differentiate into memory cells, able to provide rapid recall responses upon antigenic restimulation. Other cells will have their effector functions terminated either by anergy or by apoptotic death (which may be passive or may involve activation-induced cell death [AICD]), or as a consequence of regulation by other cells or soluble factors. CTL, cytotoxic T lymphocyte; DTH, delayed-type hypersensitivity.

interactions with other cells or with soluble factors (11). The same mechanisms act in acquired transplantation tolerance $(4,12)$ and might be harnessed to achieve donor-specific tolerance by blunting the effects of alloreactive $T$ cells. A further possible mechanism of immunologic tolerance that is unique to the transplant setting is microchimerism, the persistence of a small number of donor-derived bone marrow cells in recipients (13). However, debate continues regarding methods for detection of microchimerism, the importance of the anatomical sites in which it is found (14), as well as its clinical relevance (15). Whether tolerance or rejection is associated with microchimerism may depend on the state of maturity of the host immune system and the degree of antigenicity of the donor organ (16).

\section{Experimental induction of allograft tolerance}

Immunologic tolerance does not mean complete unresponsiveness toward the graft, but rather a lack of a destructive immune response towards it in the presence of generalized immune competence (17). How can we judge whether such a state has been achieved? Operationally, clinical tolerance is defined as absence of acute and chronic rejection and indefinite graft survival with normal graft function in an immunocompetent host. However, extensive experience with experimental animals and human patients shows that tolerance is not always permanent. Many reports claim tolerance induction after graft survival of more than 100 days in rodents, with acceptance of a second graft from the original donor strain and rejection of third-party grafts, a measure of donor-specific hyporesponsiveness. It is impossible to know how this criterion translates with regard to longevity of human transplants. Furthermore, some reports of human transplant tolerance have subsequently been revised following graft loss $(15,18)$. Accurate methods to measure such tolerance are required, so that we can monitor its induction and its breakdown, should that occur. Devising a number of different assays that allow us to prospectively follow the status of the immune response towards the graft and detect early signs of rejection or tolerance is an urgent necessity. A number of promising examples are emerging, as shown in Table 1, but their widespread clinical validation is still lacking. It is likely that a panel of assays will be required to provide an adequate immunological profile that no test could provide alone. Such assays should also allow us to make decisions regarding prospective withdrawal of immunosuppression without risking a rejection episode in tolerant patients, and detecting incipient rejection in patients during or after the withdrawal of immunosuppression.

Examples of tolerance in clinical organ transplantation exist, although most have come to light only inadvertently. Patients who have stopped their maintenance immunosuppression rarely keep their grafts, but a small number do and somehow maintain a functioning transplant (19). Other examples of patients who require no immunosuppression to maintain the transplanted organ include some who had received total body irradiation as induction therapy for their transplants $(15,18)$ and others who first received bone marrow transplants for hematological diseases and who subsequently were transplanted with kidneys from the same donor. The first such cases were published a decade ago by our group (20). More recently, Spitzer et al. published a case of a patient with multiple myeloma and renal failure that has been treated with such an approach (21).

The use of bone marrow transplantation in order to induce tolerance has been extensively studied in animal models and to a lesser extent in patients (22). Establishing mixed chimeric immune systems, with components from the donor and recipient bone marrow, allows tolerance toward the host tissues as well as the foreign graft. Interestingly, tolerance appears to outlast the chimerism in some cases, suggesting that the graft itself has some tolerogenic capacity (22). A major challenge remains to develop clinically applicable nonmyeloablative regimens that will allow bone marrow transplantation and induction of lasting chimerism and that can be safely used in HLA-mismatched patients.

An alternative approach to bone marrow chimerism involves the use of in vitro-manipulated or immature donor dendritic cells (DCs), which can induce both peripheral and central tolerance $(23,24)$. Gene targeting of transplanted organs is yet another innovative 
Table 1

Candidate outcomes to measure in a tolerance assay

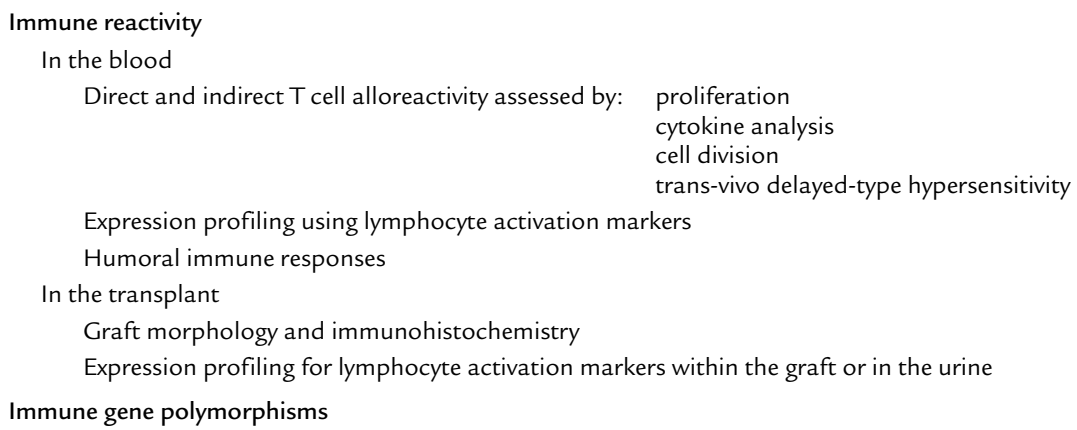

Gene chip microarrays and proteomics defining "tolerance genes" and "tolerance proteins"

relatively insensitive to other tolerance-inducing regimens.

Because of the complex interactions between $T$ cell costimulatory pathways, many regimens will need to be tested in order to establish the safety and efficacy of targeting these pathways in vivo. By analogy to the events underlying the normal tolerance to autoantigens, blockade of these costimulatory pathways may hold an alloreactive response in check only if the $\mathrm{T}$ cell population targeted is of a limited size, so that all of these cells can be tolerized. In the normal development of the immune system, most high-avidity autoreactive $T$ cell clones are elimi-

approach (25). In rodents, selective inhibition of alloimmunity has been achieved within the transplanted organ by transferring genes whose products can limit specific T cell interactions. Transfection of genes encoding immunomodulatory molecules, using adenoviral or retroviral vectors or naked DNA, can prolong graft survival in these organs but may not induce long-lasting tolerance unless used concomitantly with donor antigen-based or DC strategies (26). However, before clinical trials with either of these can be considered, evidence would be needed that nonhuman primates can be transfected safely and efficiently with antirejection genes that regulate the local graft immune response, or that manipulated DCs can be used successfully to induce tolerance. The optimal conditions and the attendant risks of these approaches must still be determined before they can be studied in humans.

There are other strategies that appear to achieve tolerance in rodent models, not all of which have yet been applied successfully in primates. The more promising strategies include the use of $\mathrm{T}$ cell costimulatory blockade $(27,28)$ or $T$ cell depleting agents $(29,30)$. Over the past several years, there has been great excitement about the potential for translating B7 and CD154 $\mathrm{T}$ cell costimulatory blockade strategies to the clinic $(10,31,32)$. Indeed, this approach has met with some success in patients with autoimmune psoriasis and in recipients of bone marrow transplants (33, 34), although it was unsuccessful in human (but not murine) systemic lupus erythematosus (35). Our understanding is still unfolding regarding how these costimulatory pathways interact with each other and with other, subdominant pathways, which appear to play major roles only under limited circumstances (36). Such pathways include those mediated by binding between ICOS and B7h, between CD134 and CD134L, between CD27 and CD70, and others (36). These have been shown to play significant roles in diverse models of autoimmunity and more recently in transplantation, as well as being found in many human diseases, in which their functions remain to be clarified. Their roles in the induction or maintenance of tolerance will require extensive investigation, since their prime action may occur in immunological compartments that are nated during ontogeny, and the small number of lower avidity cells that escape thymic selection can generally be restrained by less potent, peripheral regulatory mechanisms (37). Despite the expanded T cell clone size in autoimmune disease states, treatments that induce antigen-specific $\mathrm{T}$ cell deletion have shown some success, both in treatment of experimental animals and, to some degree, in patients (38). Similarly, reduction of the central or peripheral alloreactive $\mathrm{T}$ cell repertoire by inducing apoptosis may play a crucial role as an adjunct to treatments that aim to regulate the function of these cells (12) (Figure 2). Some of the new immunosuppressive drugs may facilitate tolerance induction in part by promoting peripheral deletion of alloreactive $\mathrm{T}$ cells (39). Limited treatment with agents such as rapamycin during the induction phase, possibly combined with $\mathrm{T}$

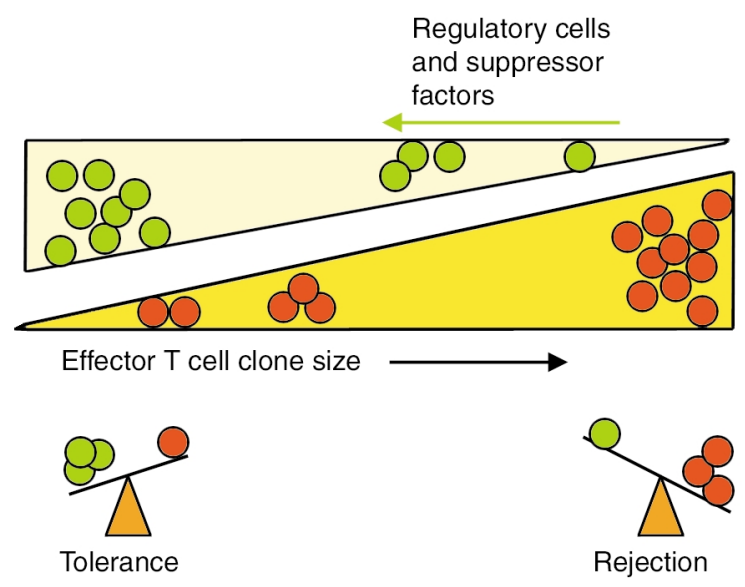

Figure 2

The balance of the immune system. This cartoon represents the balance between the effector and regulatory functions of the immune system and emphasizes the role of effector clone size in dictating the outcome of an immune response. In the presence of large numbers of effector cells (red circles), the regulatory mechanisms, consisting of regulatory cells (green circles) and suppressive soluble factors such as cytokines, are unable to attenuate the effector's response, which therefore predominates. In the context of transplantation this results in rejection. However, in the presence of low effector cell numbers these mechanisms can regulate the effectors and keep them in check. If these responses are sufficiently robust, transplant tolerance may result. Adapted from ref. 12. 
Table 2

Clinical tolerance strategies to be tested ${ }^{A}$

1. Mixed allogeneic chimerism (using bone marrow or bone marrow stem cell transplantation)

2. T cell costimulatory blockade, with or without donor antigen (bone marrow, bone marrow stem cells, or donor-specific transfusions)

3. Nonmitogenic humanized anti-CD3

4. Profound T cell depletion (Campath-1), with or without donor antigen

${ }^{A}$ Concomitant immunosuppressive regimen and duration of therapy to be decided.

cell costimulatory blockade (39) or anti-T cell antibodies, such as humanized nonmitogenic anti-CD3 (Bernard Hering, personal communication) or Campath-1, an anti-CD52 mAb (Stuart Knechtle, personal communication), may thus enhance tolerance induction. There remains the challenge of defining how much deletion is enough and how safe that is in humans. For example, Campath-1, when used in the treatment of multiple sclerosis, has been associated with the development of high rates of autoimmune thyroid disease, perhaps as a result of perturbations in the regulatory lymphocyte populations (40). Finally, whether coadministration of donor antigen in the form of donor blood transfusions or bone marrow infusion with $\mathrm{T}$ cell costimulatory blockade or $\mathrm{T}$ cell-depleting agents promotes induction of tolerance requires further testing in primates.

The contribution of conventional immunosuppressive drugs to tolerizing strategies needs to be investigated thoroughly; early fears that these drugs would impair the generation of tolerance have been borne out in some $(28,39,41)$ but not all $(42)$ models. For example, calcineurin inhibitors may facilitate tolerance induced by mixed allogeneic chimerism (42) and do not impair long-term graft survival if given with certain costimulatory blockade regimens, such as multiple rather than single doses of anti-CD154 (43). The optimal treatment strategies remain far from clear at the present time. Another important issue to consider is the timing of immunosuppression withdrawal. When a multidrug regimen is being used, this question becomes all the more complex: Which drugs are to be tapered off and at what time after transplant? Therefore, developing optimal criteria based on sound clini$\mathrm{cal}$ and laboratory studies to define tolerant patients is critical. These criteria are likely to be dependent on the tolerance regimen, such as detection of chimerism in strategies involving bone marrow or stem cell transplantation. Moreover, it is equally important to develop sensitive and reproducible assays to detect incipient rejection to monitor patients during or after withdrawal of immunosuppressive drugs (Table 1).

When thinking about developing tolerance strategies it is important to realize that what applies to rodents is often not applicable to the larger animals or to patients. In the autoimmune field, similarly, therapies such as costimulatory blockade, altered peptide ligands, and oral tolerance, which are effective in animal models, have proved disappointing or harmful in clinical trials (35). Conversely, regimens well established in clinical transplantation may be too toxic or not applicable for nonhuman primates. Therefore, in the absence of clear data in large animals, we have to adapt the strategies we already have in the clinic to include variations suggested by the animal models. However, we should accept data only from stringent small animal models that are adequately mismatched, so as to be more representative of clinical transplantation. Furthermore, the organs or tissues transplanted have to be considered, since they have varying susceptibility to tolerance induction, with liver being the easiest to tolerize, followed by kidney, heart, islets, pancreas, small bowel, and finally skin, which is the hardest. Toxicity and safety issues will undoubtedly require testing on larger animals. Table 2 lists some of the strategies that are planned for translation to the clinic.

\section{Tolerance and clinical complications following transplantation}

Achieving tolerance would avoid the current requirements for lifelong immunosuppression and many of the associated complications. However, there remain three key issues that need to be resolved: the effect of tolerance on development of chronic rejection, the relation of tolerance with specific infections, and the risk of malignancies with tolerance strategies. According to our definition, tolerance should result in the elimination of acute rejection and prevention of chronic rejection. While this prediction has been confirmed in some animal models, it is not a universal finding $(44,45)$. Only some studies in humans have indicated that donor-specific hyporesponsiveness can be associated with protection from chronic rejection (46), perhaps because tolerance may not affect alloantigen-independent mechanisms of chronic allograft dysfunction. The literature on tolerance and chronic rejection in animals is ambiguous in this regard, since a number of studies omit details of longterm graft function or histology (5).

The effect of tolerizing regimens on the development of infectious complications is also unknown, and conversely the effect particular infections may have on the induction or maintenance of tolerance is uncertain. Indeed, certain tolerizing strategies are ineffective if performed during ongoing infectious episodes (47). Tolerizing a graft in the presence of a latent infectious agent may also allow tolerance to develop toward the latter, with results that can only be speculated on at present. However, it seems prudent that patients with certain latent or chronic infections (e.g., hepatitis B or $\mathrm{C}$ viruses, cytomegalovirus, and Epstein-Barr virus) should be excluded from initial tolerance trials. In addition, since viral infections can be transmitted with the transplanted organ (48), it is advisable to exclude organs from donors infected with these viruses.

Finally, although the risk of developing post-transplant malignancies is related to the chronic immunosuppressive burden, tolerance-inducing strategies may themselves predispose certain tissues to malignant transformation following, for example, $T$ cell depletion or whole body irradiation. Adequate follow up and optimal monitoring are thus required to insure early 
detection and therapy if malignancies do occur with certain clinical tolerance strategies.

\section{Where to begin?}

The ultimate choice of which patient population will be the first to be entered into such trials remains open to debate. Important factors to consider in patient selection include the chances of success and the consequences of failure in particular groups. For example, young children ( $<3$ years old) have the best long-term renal graft survival with a significantly different rate of chronic allograft loss (49), but children also have higher rates of liver retransplantation with worse outcome than adults, making graft failure less acceptable (50). Alternatively, older recipients, who may have developed a degree of immunological senescence, may be an easier group in which to successfully induce tolerance. Other practical decisions must be made as well, such as the choice of whether to provide transplants to high- or low-risk recipients, and, in the case of kidney transplantation, whether to employ organs from cadaveric or living sources.

Some consensus on priorities for clinical testing will need to be reached, perhaps overseen by a regulatory body consisting of a panel of experts. Such a panel should also consider the ethical issues involved in risking possible rejection from a failed tolerance protocol in an era when 1-year graft survival rates exceed $90 \%$ and few grafts are lost to rejection. Furthermore, while certain types of transplants, including liver transplants, seem to be more easily tolerized and thus are excellent candidates for study, the transplant community currently has substantial reservations about attempting tolerance protocols for organs for which no suitable mechanical replacement therapies are available in the event of organ failure. Indeed, these ethical issues may be as complex as the immunologic ones.

There is the added complication that such tolerizing strategies may not benefit the pharmaceutical companies manufacturing the immunosuppressive agents currently used, and thoughtful cooperation between the biotechnology industry and the transplant biologists will be needed to successfully achieve the necessary development of the appropriate tolerizing agents. Proper conduct and execution of the trials is essential and will need to be overseen by a suitably appointed regulatory (governmental) agency. The task of coordinating these efforts might best be met by the NIH (USA) Immune Tolerance Network (http://www.immunetolerance.org), a consortium of international investigators and research groups dedicated to devising strategies and tools to induce, maintain, and monitor tolerance following organ transplantation, as well as in autoimmune and allergic diseases.

Clinical transplant tolerance is clearly achievable in particular animal models and in a few humans. Defining the most successful of these strategies and then translating them to larger animals so as to test their suitability for the majority of patients is the next step. This will require extensive investigation to confirm the robustness and longevity as well as the safety of the tolerance-inducing regimens. If we can reproduce some of the results without inducing unacceptable complications, we may arrive at this goal, although it may seem remote.

1. Billingham, R.E., Brent, L., and Medewar, P.B. 1953. Actively acquired tolerance of foreign cells. Nature.172:603-606.

2. Denton, M.D., Magee, C.C., and Sayegh, M.H. 1999. Immunosuppressive strategies in transplantation. Lancet. 353:1083-1091.

3. Neylan, J.F., et al. 1999. The allocation of cadaver kidneys for transplantation in the United States: consensus and controversy. ASN Transplant Advisory Group. American Society of Nephrology. J. Am. Soc. Nephrol.10:2237-2243.

4. Waldmann, H. 1999. Transplantation tolerance-where do we stand? Nat. Med. 5:1245-1248.

5. Womer, K.L., Vella, J.P., and Sayegh, M.H. 2000. Chronic allograft dysfunction: mechanisms and new approaches to therapy. Semin. Nephrol. 20:126-147.

6. Ojo, A.O., et al. 2000. Mycophenolate mofetil reduces late renal allograft loss independent of acute rejection. Transplantation. 69:2405-2409.

7. Kahan, B.D. 2000. Efficacy of sirolimus compared with azathioprine for reduction of acute renal allograft rejection: a randomised multicentre study. The Rapamune US Study Group. Lancet. 356:194-202.

8. Kasiske, B.L., Chakkera, H.A., and Roel, J. 2000. Explained and unexplained ischaemic heart disease risk after renal transplantation. J. Am. Soc. Nephrol. 11:1735-1743.

9. Hariharan, S., et al. 2000. Improved graft survival after renal transplantation in the United States, 1988 to 1996. N. Engl. J. Med. 342:605-612.

10. Sayegh, M.H., and Turka, L.A. 1998. The role of T-cell costimulatory activation pathways in transplant rejection. N. Engl. J. Med. 338:1813-1821.

11. Van Parijs, L., and Abbas, A.K. 1998. Homeostasis and self-tolerance in the immune system: turning lymphocytes off. Science. 280:243-248.

12. Li, X.C., Strom, T.B., Turka, L.A., and Wells, A.D. 2001. T cell death and transplantation tolerance. Immunity. 14:407-416.

13. Starzl, T.E., et al. 1992. Cell migration, chimerism, and graft acceptance. Lancet. 339:1579-1582.

14. Noris, M., et al. 2001. Thymic microchimerism correlates with the outcome of tolerizing protocols for solid organ transplantation. J. Am. Soc. Nephrol. In press.

15. Strober, S., Benike, C., Krishnaswamy, S., Engleman, E.G., and Grumet, F.C. 2000. Clinical transplantation tolerance twelve years after prospective withdrawal of immunosuppressive drugs: studies of chimerism and anti-donor reactivity. Transplantation. 69:1549-1554.

16. Anderson, C.C., and Matzinger, P. 2001. Immunity or tolerance: opposite outcomes of microchimerism from skin grafts. Nat. Med. 7:80-87.

17. Suthanthiran, M. 1996. Transplantation tolerance: fooling mother nature. Proc. Natl. Acad. Sci. USA. 93:12072-12075.

18. Strober, S., et al. 1989. Acquired immune tolerance to cadaveric renal allografts. A study of three patients treated with total lymphoid irradiation. N. Engl. J. Med. 321:28-33.

19. Burlingham, W.J., et al. 1995. Microchimerism linked to cytotoxic T lymphocyte functional unresponsiveness (clonal anergy) in a tolerant renal transplant recipient. Transplantation. 59:1147-1155.

20. Sayegh, M.H., et al. 1991. Immunologic tolerance to renal allografts after bone marrow transplants from the same donors. Ann. Intern. Med. 114:954-955.

21. Spitzer, T.R., et al. 1999. Combined histocompatibility leukocyte antigen-matched donor bone marrow and renal transplantation for multiple myeloma with end stage renal disease: the induction of allograft tolerance through mixed lymphohematopoietic chimerism. Transplantation. 68:480-484.

22. Sykes, M. 2001. Mixed chimerism and transplant tolerance. Immunity. 14:417-424.

23. Thomson, A.W., and Lu, L. 1999. Dendritic cells as regulators of immune reactivity: implications for transplantation. Transplantation. 68:1-8.

24. Lechler, R., Ng, W.F., and Steinman, R.M. 2001. Dendritic cells in transplantation: friend or foe? Immunity. 14:357-368.

25. Benigni, A., and Remuzzi, G. 1999. Genes and molecular medicine in the future of organ transplantation. Current Opinion in Organ Transplantation. 4:65-72.

26. Wood, K.J., and Prior, T.G. 2001. Gene therapy in transplantation. Curr. Opin. Mol. Ther. 3:390-398.

27. Kenyon, N.S., et al. 1999. Long-term survival and function of intrahepatic islet allografts in rhesus monkeys treated with humanized antiCD154. Proc. Natl. Acad. Sci. USA. 96:8132-8137.

28. Kirk, A.D., et al. 1999. Treatment with humanized monoclonal antibody against CD154 prevents acute renal allograft rejection in nonhuman primates. Nat. Med. 5:686-693.

29. Knechtle, S.J., et al. 1998. Primate renal transplants using immunotoxin. Surgery. 124:438-446.

30. Contreras, J.L., et al. 1999. Tolerability and side effects of anti-CD3immunotoxin in preclinical testing in kidney and pancreatic islet trans- 
plant recipients. Transplantation. 68:215-219.

31. Sayegh, M.H. 1999. Finally, CTLA4Ig graduates to the clinic. J. Clin. Invest. 103:1223-1225.

32. Kishimoto, K., Dong, V.M., and Sayegh, M.H. 2000. The role of costimulatory molecules as targets for new immunosuppressives in transplantation. Curr. Opin. Urol. 10:57-62.

33. Abrams, J.R., et al. 1999. CTLA4Ig-mediated blockade of T-cell costimulation in patients with psoriasis vulgaris. J. Clin. Invest.103:1243-1252

34. Guinan, E.C., et al. 1999. Transplantation of anergic histoincompatible bone marrow allografts. N. Engl.J. Med. 340:1704-1714.

35. Davidson, A., and Diamond, B. 2001. Autoimmune diseases. N. Engl. J Med. 345:340-350.

36. Watts, T.H., and DeBenedette, M.A. 1999. T cell co-stimulatory molecules other than CD28. Curr. Opin. Immunol. 11:286-293.

37. Anderton, S., Burkhart, C., Metzler, B., and Wraith, D. 1999. Mechanisms of central and peripheral T-cell tolerance: lessons from experimental models of multiple sclerosis. Immunol. Rev. 169:123-137.

38. Medaer, R., Stinissen, P., Truyen, L., Raus, J., and Zhang, J. 1995. Depletion of myelin-basic-protein autoreactive $\mathrm{T}$ cells by $\mathrm{T}$-cell vaccination: pilot trial in multiple sclerosis. Lancet. 346:807-808.

39. Li, Y., et al. 1999. Blocking both signal 1 and signal 2 of T-cell activation prevents apoptosis of alloreactive $\mathrm{T}$ cells and induction of peripheral allograft tolerance. Nat Med. 5:1298-1302.

40. Coles, A.J., et al. 1999. Pulsed monoclonal antibody treatment and autoimmune thyroid disease in multiple sclerosis. Lancet. 354:1691-1695.
41. Larsen, C.P., et al. 1996. Long-term acceptance of skin and cardiac allografts after blocking CD40 and CD28 pathways. Nature. 381:434-438.

42. Kawai, T., et al. 1995. Mixed allogeneic chimerism and renal allograft tolerance in cynomolgus monkeys. Transplantation. 59:256-262.

43. Sho, M., et al. 2001. New insights into the interaction between $\mathrm{T}$ cell costimulation blockade and conventional immunosuppression in vivo. American Journal of Transplantation. 1:142A. (Abstr.)

44. Hancock, W.W., Buelow, R., Sayegh, M.H., and Turka, L.A. 1998. Antibody-induced transplant arteriosclerosis is prevented by graft expression of anti-oxidant and anti-apoptotic genes. Nat. Med. 4:1392-1396.

45. Shimizu, K., Schonbeck, U., Mach, F., Libby, P., and Mitchell, R.N. 2000 Host CD40 ligand deficiency induces long-term allograft survival and donor-specific tolerance in mouse cardiac transplantation but does not prevent graft arteriosclerosis. J. Immunol. 165:3506-3518.

46. Reinsmoen, N.L., and Matas, A.J. 1993. Evidence that improved late renal transplant outcome correlates with the development of in vitro donor antigen-specific hyporeactivity. Transplantation. 55:1017-1023.

47. Turgeon, N.A., et al. 2000. Viral infection abrogates CD8(+) T-cell deletion induced by costimulation blockade. J. Surg. Res. 93:63-69.

48. Fishman, J.A., and Rubin, R.H. 1998. Infection in organ-transplant recipients. N. Engl. J. Med. 338:1741-1751.

49. Cecka, J.M., Gjertson, D.W., and Terasaki, P.I. 1997. Pediatric renal transplantation: a review of the UNOS data. United Network for Organ Sharing. Pediatr. Transplant. 1:55-64.

50. Sieders, E., et al. 2001. Retransplantation of the liver in children. Transplantation. 71:90-95. 